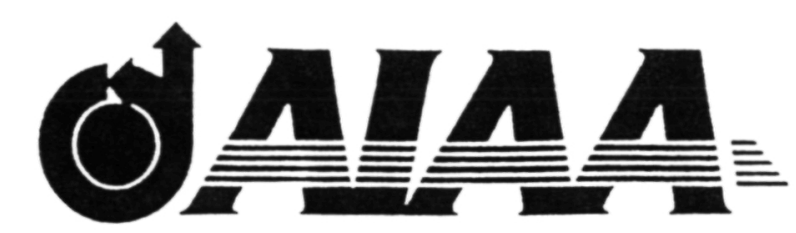

AIAA 94-0491

\title{
VOLUMETRIC IMAGING OF SUPERSONIC BOUNDARY LAYERS USING FILTERED RAYLEIGH SCATTERING BACKGROUND SUPPRESSION
}

J.N. Forkey, W.R. Lempert, S.M. Bogdonoff, and R.B. Miles

PRINCETON UNIVERSITY

Dept. of Mechanical \& Aerospace Engineering Princeton, New Jersey 08544 U.S.A.

and

G. Russell

IBM THOMAS J. WATSON RESEARCH CENTER Yorktown Heights, New York 10598 U.S.A.

\section{2nd Aerospace Sciences Meeting \& Exhibit January 10-13, 1994 / Reno, NV}




\title{
VOLUMETRIC IMAGING OF SUPERSONIC BOUNDARY LAYERS USING FILTERED RAYLEIGH SCATTERING BACKGROUND SUPPRESSION
}

\author{
J.N. Forkey, * W.R. Lempert, ${ }^{* *}$ S.M. Bogdonoff, ${ }^{\dagger}$ and R.B. Miles ${ }^{\dagger \dagger}$ \\ Dept. of Mechanical \& Aerospace Engineering \\ PRINCETON UNIVERSITY \\ Princeton, New Jersey 08544 U.S.A. \\ 609/258-5131
}

and

G. Russell

IBM THOMAS J. WATSON RESEARCH CENTER

Yorktown Heights, New York 10598 U.S.A.

\begin{abstract}
We demonstrate the use of Filtered Rayleigh Scattering and a $3 \mathrm{D}$ reconstruction technique to interrogate the highly three dimensional flow field inside of a supersonic inlet model. A 3 inch by 3 inch by 2.5 inch volume is reconstructed yielding $3 \mathrm{D}$ visualizations of the crossing shock waves and of the boundary layer. In this paper we discuss the details of the techniques used, and present the reconstructed 3D images.

\section{INTRODUCTION}

The structure of the boundary layers and crossing shock waves in supersonic inlets is a critical factor affecting engine performance, wall loading, and heat transfer to the inlet surface. In addition, the role of the boundary layer in enhancing mixing phenomena and its effect on combustion dynamics are potentially important factors for the development and optimization of supersonic RAMJET engines.
\end{abstract}

*Graduate Student, Mechanical \& Aerospace Engineering, Student Member AIAA

**Research Scientist, Mechanical \& Aerospace Engineering, Member ALAA

†Professor Emeritus, Mechanical \& Aerospace Eng., Fellow AIAA

††Professor, Mechanical \& Aerospace Engineering, Senior Member, AIAA

Copyright $@ 1994$ by the American Institute of Aeronautics and Astronautics, Inc. All rights reserved.
While a number of laser-based diagnostic techniques are available for investigating flow properties away from surfaces in supersonic and hypersonic flows, many of these have difficulty interrogating boundary layers due to large reflections of laser light from walls and windows. When available, the information obtained from these techniques is usually displayed as data along a one-dimensional line, or across a two-dimensional plane. In many practical applications, however, information about the complex three-dimensional flow structure is needed to develop a full understanding of the flow.

In this paper, we describe the use of Filtered Rayleigh Scattering to image two-dimensional slices of the flow inside of a supersonic engine inlet model. These images are attained without artificially seeding the flow, and they extend from the floor of the inlet model (including the boundary layer) to beyond the center line. We also demonstrate the use of a reconstruction algorithm to generate three-dimensional, dynamic views of the space-filling data, which provides a volumetric visualization of the flow field inside the model.

\section{EXPERIMENTAL SET-UP}

The results presented below were obtained from experiments performed in the Princeton Gas Dynamics 8 " by 8 ", high Reynolds number, Mach 3 , blowdown wind tunnel. The tunnel was run with a stagnation temperature of $260 \mathrm{~K}$, stagnation pressure of $100 \mathrm{psi}$ and with a free stream Mach number of 3. A schematic of the inlet model which was placed in the $8 " x 8^{\prime \prime}$ test section is shown in Fig. 1.1.2.3 The model consisted of two 24-inch long flat plates, each mounted parallel to the wind tunnel floor and ceiling. The bottom plate was mounted 2 inches from the floor of the wind tunnel, 
while the top plate was mounted 2 inches from the ceiling of the wind tunnel (4 inches from the bottom plate). The boundary layer on the wind tunnel walls was approximately 1 inch thick at the leading edge of the flat plates, so the flow entering the model was free of any initial boundary layer (i.e., the top and bottom boundary layers inside the model were built-up starting from the leading edge). Between these two flat plates, were two variable angle vertical fins. The leading edges of the fins were separated by approximately 6 inches, and were 7.6 inches downstream of the leading edges of the flat plates. Although each fin angle could be varied independently from 5 degrees to 11 degrees with respect to the wind tunnel free stream flow direction, all of the data reported in this paper were taken with both fins set at 11 degrees. Optical access to the inlet model was provided by BK7 and quartz windows mounted in the floor and ceiling of the wind tunnel and in the two flat plates in the model.

In order to image the boundary layer close to the bottom flat plate, Filtered Rayleigh Scattering (FRS) was used. ${ }^{4,5,6}$ The laser source was a frequency-doubled, injection-seeded, Nd:YAG laser which generated $5 \mathrm{~ns}$ pulses, each with $300 \mathrm{~mJ}$ of energy at a wavelength of $532 \mathrm{~nm}$. The laser light was focused into a sheet $0.8 \mathrm{~mm}$ thick and $7 \mathrm{~cm}$ wide and passed through the model oriented perpendicular to the flat plates, and spanwise with respect to the incident free stream flow (see Fig. 2). Light from the laser sheet was scattered by an ice vapor fog which is formed in the cold regions of the flow from a few parts per million residual water vapor in the compressed air supply. Other investigators have used larger amounts of water vapor or have seeded their flows with ethanol. ${ }^{7.8 .9}$ However, in our case, the FRS technique was sensitive enough that the residual concentration of water vapor was sufficient to obtain images. While scattering from this ice vapor fog precludes the possibility of observing molecular Rayleigh scattering, it provides a means of generating images with large contrast between "warm" and "cold" regions of the flow. In particular, interfaces across shocks, and interfaces between boundary layers and free stream flow are accentuated.

The light scattered from the flow in the downstream direction, was turned by a $3 \frac{1}{8}$ inch by $1 \frac{5}{8}$ inch, front surface aluminum mirror which was placed roughly 27 inches downstream of the leading edges of the flat plates. This mirror was mounted at an angle of 45 degrees to the tunnel walls, on the end of a sting arm. Light reflected from this mirror passed through a quartz window mounted in the side of the tunnel and was imaged by a Vivitar zoom lens, onto a double-intensified CID camera. The camera output was stored on videotape for subsequent analysis.

Images were taken at 26 different downstream locations inside of the model, with the farthest upstream location 12.3 inches downstream of the leading edges of the flat plates. Subsequent locations were separated from each other by 0.1 inches. Each image captured a slice of the flow roughly 3 inches high and 3 inches wide. In order to preserve the camera magnification as the laser sheet was translated, the camera assembly was also translated in 0.1 inch increments.

Filtering of the scattered light was accomplished by placing a glass cell filled with iodine vapor in front of the camera lens. The cell was 3.25 inches long. The cell temperature was held at $80 \mathrm{C}$, and the vapor pressure was held at 1.44 torr by keeping the temperature of a cooler side arm at $44.5 \mathrm{C}$. An absorption profile for this cell in the frequency region of interest has been measured previously ${ }^{5}$ and is shown in Fig. 3. By tuning the frequency of the injection seeded laser to overlap the strong absorption feature labeled A in Fig. 3, much of the stray scattering from walls and windows was absorbed by the iodine cell before it reached the camera since this stray light was at the same frequency as the incident laser light. Light scattered from the flow, however, was Doppler-shifted to a frequency outside of absorption feature A (see Fig. 3), and so this light passed through the iodine cell and was imaged onto the camera. Because the viewing angle in this experiment was 180 degrees with respect to the free stream velocity, and 90 degrees with respect to the illumination source, the Doppler shift due to the flow velocity was large (on the order of $1 \mathrm{GHz}$ ), so this experiment was an ideal application of the Filtered Rayleigh Scattering technique. In order to illustrate the utility of Filtered Rayleigh Scattering in this configuration, Fig. 4 shows two images taken with the camera "zoomed in" on the upper boundary layer. Figure 4a shows the image obtained without using the vapor filter. Here it is impossible to see any of the roughly $5 \mathrm{~mm}$ thick boundary layer. Figure $4 \mathrm{~b}$ is identical to that of Fig. $4 a$ except that this image was acquired using FRS. In this case, the boundary layer is clearly visible.

\section{DATA PROCESSING}

Images were initially stored on video tape, and were subsequently processed using a Data Translation DT1451 frame-grabber installed on a Sun 4 Workstation. A single image from each of the 26 different downstream locations was used for the three-dimensional reconstruction. Because the turning mirror oscillated slightly in the horizontal direction during wind tunnel runs, each image was digitally shifted using as a reference a vertical line located half way between the two shocks generated by the two fins. Other image processing operations that were performed were a standard cropping of the image to eliminate data outside of the field of interest, and a standard greyscale stretch to increase contrast. Because the greyscale stretch was performed after digitization, the dynamic range of the final images was not as large as it would have been if the gain on the camera amplifier had been set higher, or if an analog amplifier had been used when the images were 
transferted from the VCR to the frame grabber. We have incorporated such an amplifier into the system for future experiments. Finally, strong, spurious noise signals, which were generated by scattering of laser light by large particles in the flow, were artificially removed from some images when the area surrounding these signals was uniformly black. These large particles, probably "large" ice crystals, are observed near the end of each wind tunnel run. Given a sufficient number of wind tunnel runs, it would have been possible to limit run times so as to avoid generating these particles. However, due to wind tunnel time constraints, this was not done.

The processed two-dimensional images are shown in Fig. 5. In the first 11 images, the edge of the turbulent boundary layer on the bottom flat plate is clearly visible. Outside of the boundary layer, where the static temperature is cold, the ice vapor fog is present, and much light is scattered. Within the boundary layer, however, the static temperature is much higher, so the ice vapor fog disappears, and very little light is scattered. The interface between the dark and light regions, therefore, indicates the location of the gradient in static temperature at the edge of the boundary layer. The effect on the boundary layer, of the strong pressure gradients generated across the vertical shocks is clearly seen in these images. Boundary layer fluid is forced to the centerline of the model causing a "bulging" of the boundary layer as the flow moves downstream.

Shock locations in these images are also clearly visible. In the first 7 images taken at locations ahead of the shock crossing point, the shocks are observed as curved lines of slightly increased intensity extending from the top of the field of view to the bottom boundary layer. In the images taken farther downstream, beyond the shock crossing point, the static temperature after the shocks is high enough that the ice vapor fog disappears completely, and so shocks are observed here as sharp discontinuities between bright and dark regions of the images. The two crossing shocks observed in these images exhibit a continuous curvature from the bottom boundary layer to the top of the field of view. This is in contrast to the shocks observed by Garrison and Settles et. al. inside an inlet model without a top flat plate. ${ }^{9}$ In this latter case, shocks were observed to be curved close to the boundary layer, but straight and vertical farther from the bottom plate.

\section{THREE-DIMENSIONAL RECONSTRUCTION}

In order to increase our understanding of the evolution of the shocks and boundary layer as they move through the inlet model, we have utilized a display method which was developed for viewing translucent 3D space-filling data. ${ }^{10,11}$ This method projects the 3-D space-filling data onto a 2-D screen (the computer screen) which is located at some specified distance from the center of the 3-D volume, and at an angle determined by the specified observer location (see Fig. 6). The intensity of each pixel on the computer screen is determined by tracing rays from the back of the data set to the observer's location, through all of the data slices. At each intersection point between the ray and an individual volume element (voxel), the "incoming intensity," generated from voxels farther from the observer is first attenuated by a value determined by the voxel data value and then incremented by an amount proportional to the data value. This method creates an image with "partial transparency" where, as in viewing fog, data closer to the observer partially obscures data farther back. Mathematically, this can be expressed as:

$$
\mathrm{I}_{\text {out }}=\mathrm{I}_{\text {in }} * \exp (-\alpha \mathrm{L})+\mathrm{I}_{\text {local }}
$$

where $\mathrm{I}_{\mathrm{in}}$ is the intensity generated by previous data slices; Ilocal is the local source intensity--a function of data value; $\alpha$ is the local absorption coefficient--also a function of data value; and $\mathrm{L}$ is the distance between data slices. This technique is capable of projecting onto a 2-D screen not only solid surfaces, but also, because of the partial transparency, objects embedded within the 3-D volume.

This technique was used to generate 2-D projections of the 3-D flow in the inlet model at a number of different viewing locations. All of the viewing angles placed the observer slightly above the inlet model, looking down at an angle of 30 degrees. Different viewing locations varied as the observation angle was rotated about the imaginary vertical axis passing through the center of the inlet model. This angle was varied from 0 degrees to 360 degrees in three degree increments. Examples of the resulting 2-D projections are shown in Fig. 7.

These reconstructions provide a way to view all of the data in one 2-D image. While individual projections yield some insight into the three dimensionality of this flow, two additional techniques further enhance the perceived three-dimensionality. By rapidly displaying images reconstructed with successive observation angles (each separated by three degrees from the previous one), the data is observed as a rotating volume. This rotation greatly increases the perception of three dimensionality by allowing the observer to perceive relative locations of different portions of the data. This dynamic approach simulates the way one naturally observes a complex volumetric object by moving one's head.

Because it is not always possible to rapidly redisplay the reconstructed images, particularly in a manuscript, we have also investigated the use of stereo pairs of images. The image shown in Fig. $7 \mathrm{~b}$ is redisplayed in Fig. 8, along with its associated stereo pair. The viewing of such stereo pairs can be aided by coloring one of the images blue, and adding to it the second image colored red. Then the three-dimensionality is perceived by viewing this composite picture through glasses with one red lens and one blue lens. Although this multi-color scheme aids in the viewing of stereo 
pairs, we have found that the use of apparent motion is, by far, the better method for understanding the threedimensionality of space-filling data. For this particular data set, the use of this dynamic technique allowed for a clearer understanding of the three-dimensional shape of the shocks. Particularly noticeable were the curvature of the shocks, as well as the shape of their approach towards and separation after the crossing point.

In order to focus in on the 3-D shape of the boundary layer, the first 11 images, which show a well defined boundary layer edge, were cropped just above the boundary layer. Also, the greyscale values were inverted in order to highlight the boundary layer. These cropped and inverted image slices were then used to generate the 3-D reconstructions as described above. Once again, rotating images were generated and were used to observe the "central bulging" of the boundary layer as it moved downstream. A stereoscopic pair of images showing this effect is shown in Fig. 9.

\section{FUTURE WORK}

Future efforts will be aimed at achieving two goals. The first is to image molecular Rayleigh scattering in this inlet model. This will allow for the investigation of the flow farther downstream where the flow is too warm to support the ice vapor fog. In addition, it will allow for the investigation of this entire flow, when run with pure nitrogen. Preliminary results were obtained using the setup described above with the laser sheet moved to a point 16 inches downstream of the leading edges of the flat plates: a location where the ice fog no longer appears. With no further optimization of laser seeding efficiency, iodine filter characteristics, or collection geometry, the time averaged image shown in Fig. 10b was obtained. Because of motion of the turning mirror, edges in this image are not very sharp. However, this image shows that the variation in intensity across the edge of the boundary layer can be detected with this FRS technique. By contrast, a similar image was taken with the FRS technique disabled. This image is shown in Fig. 10a. In this case, stray wall scattering completely overwhelms the Rayleigh scattering.

The second major area under investigation is the further development of FRS to be able to make quantitative measurements of temperature, density and velocity. Early work in this area has already been reported. ${ }^{5}$ In this work, we found that one of the major sources of systematic uncertainty is due to uncertainty in laser frequency. Currently under development is a system which accurately measures laser frequency by measuring the beat frequency between the interrogation laser and a reference laser locked to a well characterized iodine absorption line. We expect that this system will be able to measure laser frequency to an accuracy on the order of $1 \mathrm{MHz}$. This corresponds to a velocity uncertainty, for example, on the order of $1 \mathrm{~m} / \mathrm{s}$.

\section{CONCLUSIONS}

In this work, we have demonstrated the utility of the Filtered Rayleigh Scattering technique for investigating complex supersonic flows. In particular, FRS is especially useful when imaging boundary layers close to surfaces, where stray scattering would otherwise be a problem. We have also demonstrated the utility of a 3D reconstruction technique, which, when used in a dynamic mode, can generate the perception of a rotating volume of data. Alternatively, stereo pairs of images can be used to generate 3D perception.

Finally, we expect that future work will allow for the imaging of molecular Rayleigh scattering, and will generate quantitative data with lower levels of experimental uncertainty.

\section{ACKNOWLEDGMENTS}

We wish to acknowledge Carl Delo and Jon Poggie for their help in generating the 3D reconstructions. Also, we would like to acknowledge Bill Stokes for his help in setting up and running the wind tunnel experiments.

This research was conducted with support from the AFOSR and NASA-Langley. In addition, J. Forkey was supported by a graduate student researchers fellowship from NASA-Lewis.

\section{REFERENCES}

1. K. Poddar and S. Bogdonoff, "A Study of Unsteadiness of Crossing Shock Wave Turbulent Boundary Layer Interactions," AIAA \#90-1456, 21st Fluid Dynamics, Plasma Dynamics and Lasers Conference, Seattle, WA, June 18-20, 1990.

2. N. Narayanswami, D. Knight, S.M. Bogdonoff, C.C. Horstman, "Crossing Shock Wave--Turbulent Boundary Layer Interactions," AIAA 91-0649, 29th Aerospace Sciences Meting, Reno, NV, Jan. 7-10, 1991.

3. S.M. Bogdonoff and W.L. Stokes, "Crossing Shock Wave Turbulent Boundary Layer Interactions--Variable Angle and Shock Generator Length Geometry Effects at Mach 3," AIAA 92-0636, 30th Aerospace Sciences Mtg. Exhibit, Reno, NV, Jan. 6-9, 1992.

4. R.B. Miles, W.R. Lempert, and J. Forkey, "Instantaneous Velocity Fields and Background Suppression by Filtered Rayleigh Scattering," AIAA 910357, 29th Aerospace Sciences Mtg., Reno, NV, Jan. 79, 1991.

5. R.B. Miles, J.N. Forkey, and W.R. Lempert, "Filtered Rayleigh Scattering Measurements in Supersonic/Hypersonic Facilities," AIAA 92-3894, 7th Aerospace Ground Testing Conf., Nashville, TN, July 68, 1992. 
6. G.S. Elliott, M. Samimy, and S.A. Arnette, "Molecular Filter-Based Diagnostics in High-Speed Flows," AIAA 93-0512, 31st Aerospace Sciences Meeting and Exhibit, Reno, NV, Jan. 11-14, 1993.

7. G.S. Elliott, M. Samimy, and S.A. Arnette, "A Study of Compressible Mixing Layers Using Filtered Rayleigh Scattering," AIAA 92-0175, 30th Aerospace Sciences Meeting and Exhibit. Reno, NV, Jan. 6-9, 1992.

8. F.S. Alvi and G.S. Settles, "Physical Model of the Swept Shock Wave/Boundary Layer Interaction Flow Field," AIAA Journal 30, Sept. 1992, p. 2252.

9. T.J. Garrison and G.S. Settles, "Flow Field Visualization of Crossing Shock Wave/Boundary Layer Interactions," 30th Aerospace Sciences Meeting and Exhibit, Reno, NV, Jan. 6-9, 1992.

10. G. Russell and R.B. Miles, "Display and Perception of 3-D Space-Filling Data," Applied Optics 26, March 15, 1987, p. 973.

11. C. Delo, J. Poggie, and A.J. Smits, "A System for Imaging and Displaying Three-Dimensional, TimeEvolving Passive Scalar Concentration Fields in Fluid Flow," Princeton University MAE Dept. Technical Repor \#1992, Jan. 1994.

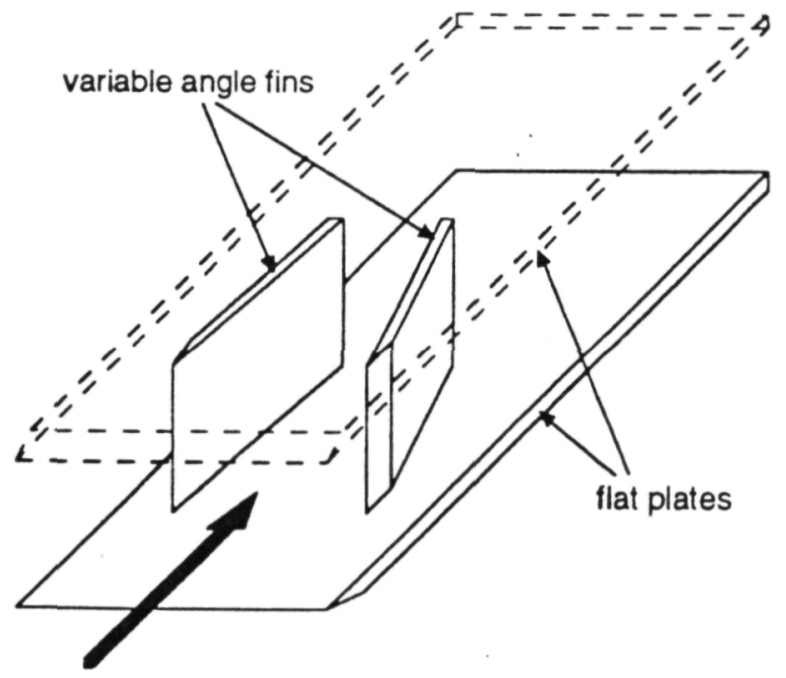

Fig. 1) Experimental model of supersonic inlet. Flat plates are separated by 4 inches. Leading edges of fins are separated by 6 inches.

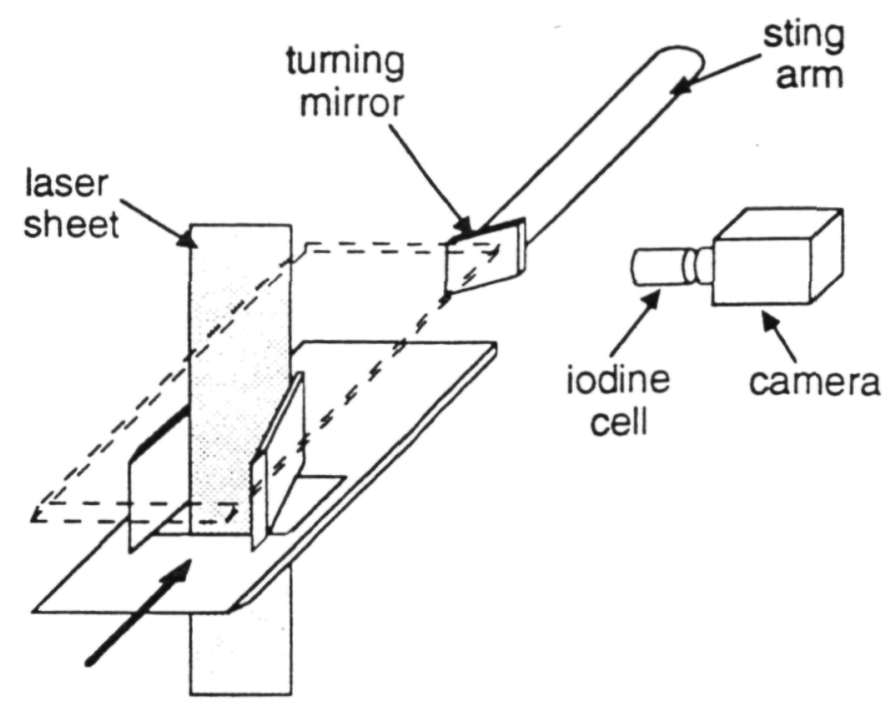

Fig. 2) Experimental setup for Filtered Rayleigh Scattering technique.

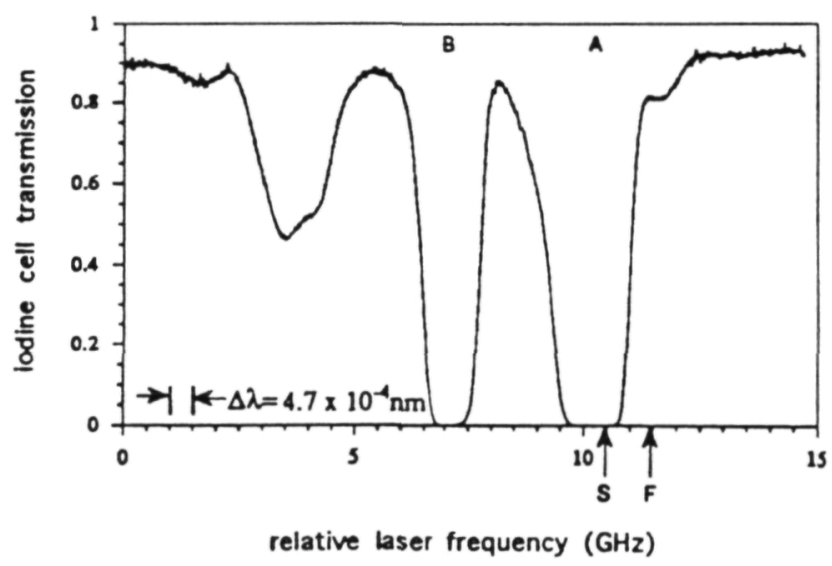

Fig. 3) Absorption profile of iodine cell near $532 \mathrm{~nm}$. The absorption feature labelled " $A$ " was used as the filter in the work reported here. Stray scattering was at the frequency indicated by " $S$ ", while scattering from the free stream of the flow was at the frequency indicated by "F". 
Fig. 4) Images of the boundary layer on the top flat plate a) taken without using FRS, b) taken with FRS.

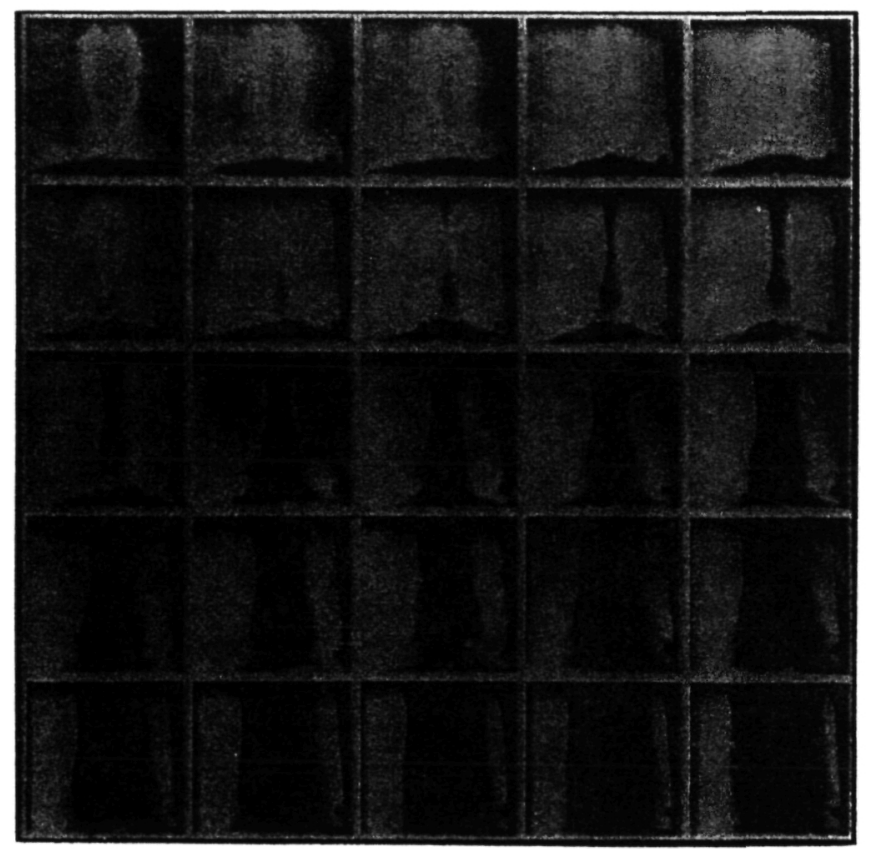

(Fig. 7a)

Fig. 5) 3 inch by 3 inch slices of data showing shock structure and bottom plate boundary layer. The image on the top left was taken 12.4 inches downstream of the leading edge of the flat plate. Subsequent images were seperated from each other by 0.1 inches.

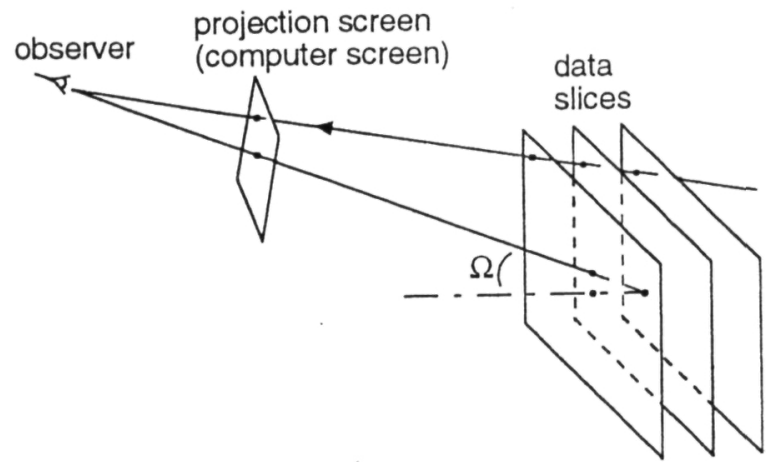

Fig. 6) Geometry used in 3D reconstruction technique. 

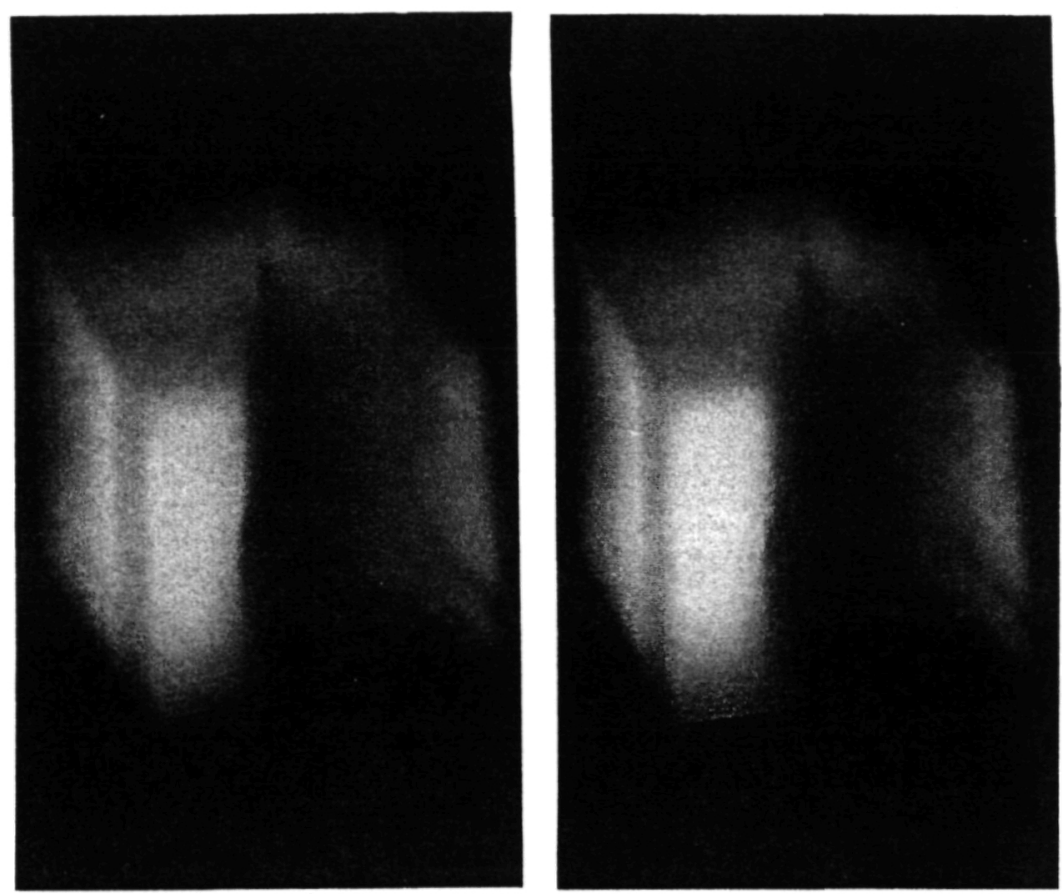

Fig. 8) This is the image in figure $7 \mathrm{~b}$ redisplayed with its stereopair.
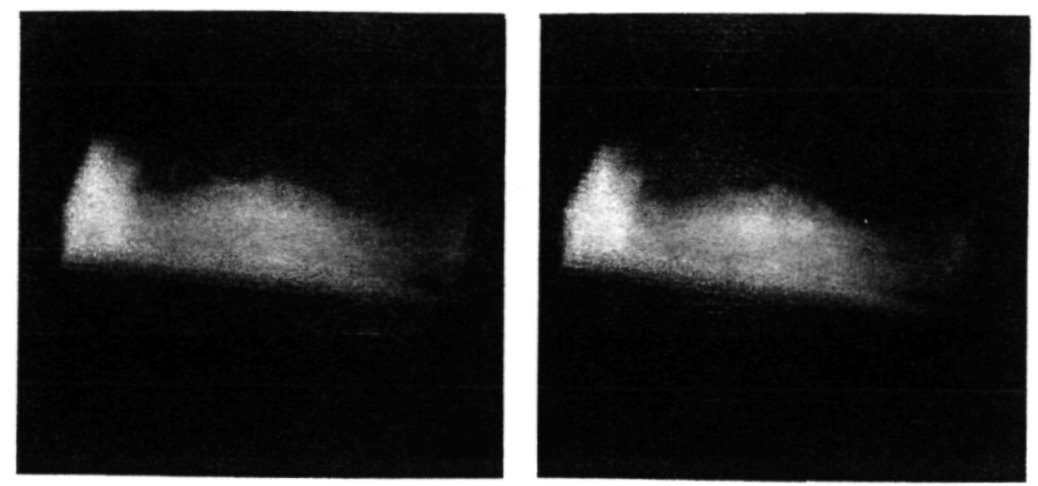

Fig. 9) Stereopair of boundary layer as seen by an observer looking downstream.

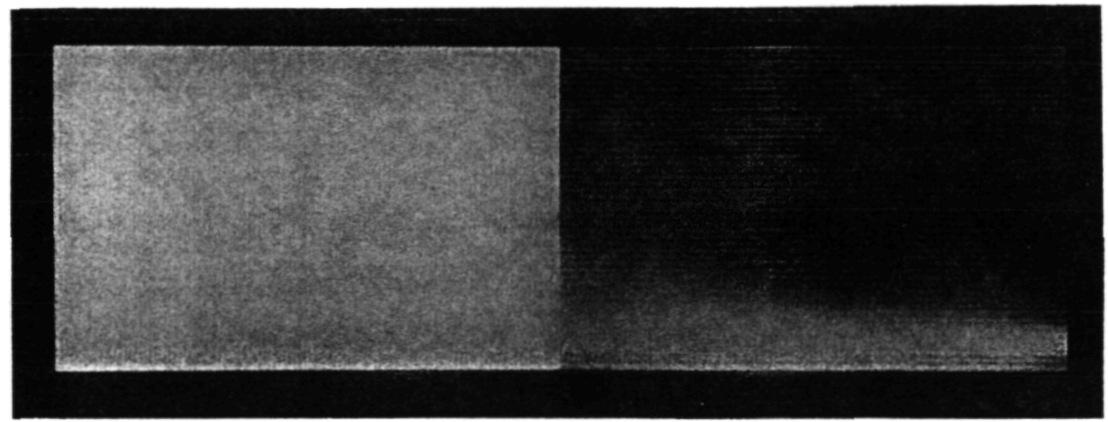

(Fig. 10a)

(Fig. 10b)

Fig. 10) Images of molecular Rayleigh scattering taken 16 inches downstream of the leading edges of the flitt. platcs, it point where ice vapor is not present. a) Image taken with no filtering. b) Image taken using Filtered Raylcigh Scattering, showing semicircular boundary layer. 